\title{
DESEMPEÑO DEL DOCENTE INSTRUMENTADOR EN EL CAMPO DE PRÁCTICA DESDE LA
} PERSPECTIVA DE LOS ESTUDIANTES

\section{PERFORMANCE OF THE TEACHING INSTRUMENTATOR IN THE FIELD OF PRACTICE FROM THE PERSPECTIVE OF THE STUDENTS}

\section{Marcela AQUINO'1.}

${ }^{1}$ Universidad Nacional de Asunción, Facultad de Ciencias Médicas, San Lorenzo, Paraguay.

Cómo citar este artículo: Aquino M. Desempeño del docente instrumentador en el campo de la práctica desde la perspectiva de los estudiantes. Med. clín. soc. 2019;3(1):9-13.

\section{RESUMEN}

Introducción: Reconocer el desempeño del docente instrumentador/a en el campo de práctica, desde la perspectiva de los estudiantes. Objetivo: Analizar el desempeño del docente instrumentador/a en el campo de práctica, desde la perspectiva de los estudiantes de la Carrera de Instrumentación y Área Quirúrgica de la Facultad de Ciencias Médicas de la Universidad Nacional de Asunción. Metodología: El nivel de la investigación científica se ubicó en descriptivo, de corte transversal, dado que se realizó una encuesta para la recolección de la información de fuente primaria y análisis con métodos descriptivos. Con enfoque cuantitativo. Resultados: El $90 \%$ de los encuestados indica que el docente debe acompañar en Todas las áreas de desempeño. El desempeño docente ha sido calificado por parte de los encuestados, con un $44 \%$ que ha indicado Bueno. Encontrándose que tanto el criterio Excelente con $16 \%$, Muy buena $24 \%$, sumados estas tres cifras numéricas nos da un $84 \%$ de gestión positiva para la formación de profesionales, frente a un $16 \%$ que se ubica en la categoría de Regular. Discusión: Es indispensable que el docente instrumentador esté preparado para poder brindar solución al estudiante y respuesta ante un interrogante al menos intentar disipar las dudas y bridar la respuesta que están esperando.

Palabras clave: docente; instrumentador.

\section{ABSTRACT}

Introduction: Recognize the performance of the instrumentation teacher in the field of practice, from the perspective of the students. Objective: To analyze the performance of the instrumentation teacher in the field of practice, from the perspective of the students of the Instrumentation and Surgical Area of the Faculty of Medical Sciences of the National University of Asunción. Methodology: The level of scientific research was descriptive, of a cross-sectional nature, since a survey was carried out for the collection of primary source information and analysis with descriptive methods. With a quantitative approach Results: $90 \%$ of respondents indicate that the teacher must accompany in all areas of performance. The docent performance has been rated by the respondents, with $44 \%$ saying Good. Finding that both the Excellent criterion with $16 \%$, Very good $24 \%$, added these three numerical figures gives us $84 \%$ positive management for the training of professionals, compared to $16 \%$ that is located in the Regular category. Discussion: It is essential that the instrumentation teacher be prepared to be able to provide a solution to the student and answer a question at least try to dispel the doubts and bridle the answer they are expecting.

Keywords: teacher; instrumentation 


\section{INTRODUCCIÓN}

La Carrera de Instrumentación y Área Quirúrgica es una carrera de grado que pretende obtener un egresado con formación teórico/práctico, en los diversos campos relacionados con las áreas de competencias.

La actividad del docente, en este contexto se trasforma en un elemento importante en el proceso, ya que, al integrarse con los estudiantes en las diferentes áreas del ambiente quirúrgico, en el campo de prácticas proporciona a través de sus conocimientos actuales las bases y el apoyo necesario para el desarrollo del futuro profesional que se centre en la excelencia y cuyo objetivo primordial y final es la seguridad del paciente quirúrgico.

La actuación del docente instrumentador, frente a sus metas, objetivos y resultados deben ser evaluados, lo cual permitirá definir el índice de gestión. La evaluación del desempeño docente en la educación superior se ha realizado por diversos procedimientos y métodos, tales como: la autoevaluación del profesor, la evaluación por pares y responsables académicos y la evaluación por los estudiantes, por solo citar algunos de los procedimientos más difundidos (1).

Valdés comenta que la evaluación docente tiene las siguientes funciones: a) diagnóstica, b) instructiva, c) educativa y d) desarrolladora. Además, propone una serie de modelos, entre los que se encuentran los siguientes: - Centrado en el perfil de profesor ideal. - Centrado en los resultados alcanzados por sus alumnos. $\bullet$ Centrado en el comportamiento del docente que se relaciona con los logros de los alumnos (2).

La tarea del docente es enseñar, orientar a los estudiantes para que logren cambios de conducta y al mismo tiempo educar, es decir contribuir al logro de la finalidad de la educación (3). La evaluación del desempeño docente por estudiantes tiene como objetivo valorar las fortalezas y dificultades de las actividades desarrolladas por los docentes en el trabajo formativo; por su naturaleza, constituye una herramienta que proporciona información útil para adecuar las estrategias institucionales, de capacitación y desarrollo de competencias de los profesores (4). Diseñar un instrumento de evaluación que ayude al docente instrumentador, orientar las prácticas hospitalarias a la luz de las corrientes pedagógicas vigentes.

\section{METODOLOGÍA}

El nivel de la investigación científica se ubicó en descriptivo, de corte transversal, dado que se realizó una encuesta para la recolección de la información de fuente primaria y análisis con métodos descriptivos. La población enfocada son los estudiantes que se encuentran matriculados y cursando la Carrera de Instrumentación y Área Quirúrgica de la Facultad de Ciencias Médicas de la Universidad Nacional de Asunción, del periodo lectivo 2017.

Se incluyó a cada uno de los estudiantes que se encuentran matriculados y cursando el $2^{\circ}, 3^{\circ}$ y $4^{\circ}$ curso respectivamente de la carrera de Instrumentación y Área Quirúrgica de la Facultad de Ciencias Médicas de la Universidad Nacional de Asunción, del periodo académico 2017.

La muestra estuvo conformada por 50 estudiantes de la carrera de Instrumentación y Área Quirúrgica de la Facultad de Ciencias Médicas de la Universidad Nacional de Asunción, del periodo académico 2017 , distribuidos entre 18 del $2^{\circ}, 18$ del $3^{\circ}$ y 14 del $4^{\circ}$ curso. 
Como criterio de inclusión se ha tenido en cuenta que los estudiantes: Se encuentren matriculados a la Carrera de Instrumentación y Área Quirúrgica del periodo académico 2017 y presentes en las prácticas hospitalarias en el momento de la recolección de datos. Los estudiantes del 1er. curso no fueron incluidos para la muestra ya que los mismos no realizan aún práctica quirúrgica. Se respetaron los principios de la ética aplicada a investigaciones y se mantuvo el anonimato de los participantes.

\section{RESULTADOS}

Al realizarse la distribución de la muestra los estudiantes del $2^{\circ}$ y $3^{\circ}$ curso tuvieron igualdad de participación $36 \%$. A diferencia del $4^{\circ}$ curso que durante la distribución de la muestra alcanzaron el $28 \%$ de participación como encuestado.

El 90\% de los encuestados indica que el docente debe acompañar en Todas las áreas de desempeño (Quirófano, área quirúrgica y esterilización) para su formación práctica durante la carrera de Instrumentación y Área Quirúrgica. Un 10\% de los encuestados indican que el Área Quirúrgica es el lugar de interés para el acompañamiento del docente.

En lo que desempeño del docente instrumentador en el campo de práctica, desde la perspectiva de los estudiantes el desempeño docente ha sido calificado por parte de los encuestados, con un $44 \%$ que ha indicado Bueno. Encontrándose que tanto el criterio Excelente con 16\%, Muy buena $24 \%$, sumados estas tres cifras numéricas nos da un $84 \%$ de gestión positiva para la formación de profesionales, frente a un $16 \%$ que se ubica en la categoría de Regular.

FIGURA 1. DESEMPEÑO DEL DOCENTE INSTRUMENTADOR EN EL CAMPO DE PRÁCTICA, DESDE LA PERSPECTIVA DE LOS ESTUDIANTES $(n=50)$

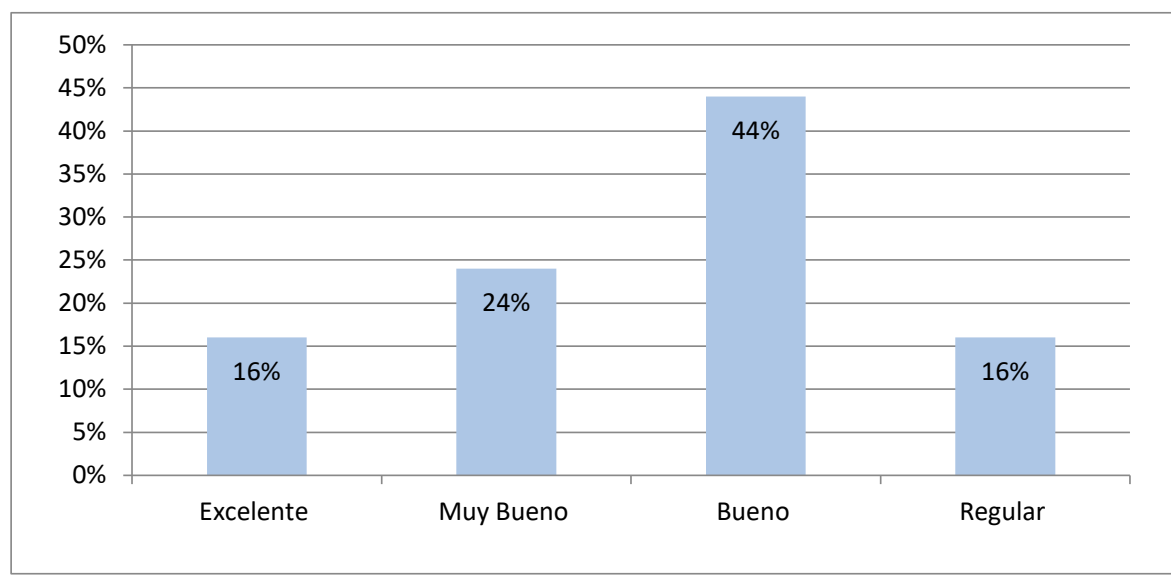

Los encuestados consideran que el aspecto que más valoran en el docente instrumentador es el Acompañamiento en las diferentes áreas de competencias durante la práctica siendo este el indicador con mayor aceptación con un $32 \%$, en un orden descendiente $28 \%$ indicó que es el Conocimiento. El 20\% indicó Buen Trato en tercer lugar, en cuarto lugar, se encuentra el Transmitir Seguridad al Estudiante y dentro del último nivel la Responsabilidad con el 6\%.

Según el $24 \%$ de los encuestados, los mismos consideran que como debilidades del docente instrumentador, son la Puntualidad y el Interés por escuchar al estudiante y ayudar ante una dificultad. El 22\% indica que el Acompañamiento durante las distintas actividades y la Entrega de planillas informativas a la coordinación con puntualidad y mantener informado al estudiante sobre su desempeño son los puntos que deberían mejorar. 
Un 8\% expresa que el docente instrumentador cuenta con una debilidad en el área de Comprensión y Equidad. El $98 \%$ afirma que le gustaría que el docente instrumentador lo oriente sobre lo realizado luego de un procedimiento u otra actividad dentro de la práctica.

Según el $86 \%$ de los encuestados han manifestado que sí afecta el desempeño del docente instrumentador la cantidad de estudiantes distribuidos por práctica, mientras que el $10 \%$ refiere que no afecta. Para el $4 \%$ no es relevante la cantidad de estudiantes.

Un promedio de 1 - 6 estudiantes por docente instrumentador es el indicado para el $82 \%$ de los encuestados. El 16\% considera que entre 7-12 estudiantes por docente sería el ideal. Y el $2 \%$ considera que entre $13-16$ estudiantes.

\section{DISCUSIÓN}

El proceso de aprender a enseñar, es necesario para comprender mejor la enseñanza y para disfrutar de ella (5). El docente de Instrumentación y área quirúrgica dentro del campo de práctica es un formador, un guía, un orientador y un estratega de los aprendizajes.

Es indispensable que el docente instrumentador esté preparado para poder brindar solución al estudiante y respuesta ante un interrogante al menos intentar disipar las dudas y bridar la respuesta que están esperando. Identificar las fortalezas y las debilidades que el alumno refiere es una forma de tomar conocimiento de algunas actitudes a tener en cuenta con el fin de siempre ir avanzando y buscar llenar así las expectativas de ellos como docente.

Muchas veces la oportunidad de que el alumno adquiera las destrezas manuales necesarias para la profesión es limitada debido a la cantidad de estudiantes para cada docente instrumentador en el campo de práctica. Una posible solución al tema podría ser la incorporación de nuevos docentes instructores como también la posibilidad de conseguir desde el inicio del periodo lectivo la incorporación de otros campos hospitalarios.

Actualmente la Escuela de Instrumentación y Área Quirúrgica cuenta con un total de 16 docentes instrumentadores, de los cuales 4 cumplen la función dentro del Pabellón Quirúrgico Central del Hospital de Clínicas, en el turno de práctica y se distribuyen por día. Los demás están cubriendo guardias dentro del pabellón quirúrgico central y otros servicios quirúrgicos dentro del Hospital de Clínicas y algunos en otros centros hospitalarios donde también los estudiantes acuden para las prácticas.

Es relevante recordar que en el año lectivo 2017 la cantidad de estudiantes que debe realizar su práctica dentro del campo quirúrgico se distribuye de la siguiente forma: Cursos: $2^{\circ}: 51$ estudiantes, $3^{\circ}: 54$ estudiantes, y $4^{\circ}: 37$ estudiantes. El quirófano como ambiente del aprendizaje esta forzado a ejercer como dispositivo de poder en su doble condición de ser una dependencia del hospital y de operar con funciones educativas, sumando a la necesidad de ordenarse bajo rigurosas prácticas de control de múltiples niveles, dando el impacto de sus actividades (6). 
Existen diferentes tipos de disposiciones adecuadas para los quirófanos modernos. Aunque estás varían de una institución a otra A causa del peligro constante de contaminación del paciente quirúrgico, los ingenieros de diseños se esfuerzan por delinear las áreas (7).

Las prácticas se van organizando a través de cronogramas como también la distribución para las guardias y para otros servicios quirúrgicos. El Pabellón Central del Hospital de Clínicas es el que más estudiantes recibe, asisten los estudiantes de los 3 cursos con un docente instrumentador responsable, ya que ahí pueden encontrar procedimientos generales y también procedimientos de especialidades como ser Vascular, Urología, Traumatología, Otorrinolaringología entre otros. Aunque como manifestaron los estudiantes encuestados las actividades de competencia en su formación profesional abarcan varias áreas y es así que el docente instrumentador busca la estrategia de poder estar y orientar en las diferentes áreas de competencia.

Es importante recalcar que siempre se cuenta con el apoyo de los estudiantes de cursos superiores que orientan en algunos casos a sus futuros colegas que están empezando. Culminando no podemos olvidar el tema de la evaluación; como aporte después de este trabajo de investigación dejo a consideración un Instrumento de Evaluación que puede ser útil para el docente instrumentador durante la práctica y/o guardia, que está basado en las competencias de importancia durante la formación práctica del futuro profesional, un tema que han manifestado en un $96 \%$ de importancia para ellos.

\section{CONFLICTOS DE INTERÉS Y FUENTE DE FINANCIACIÓN}

La autora declara no poseer conflictos de interés. Fuente de financiación: ninguna.

\section{REFERENCIAS BIBLIOGRÁFICAS}

1. Tobón S, Pimienta Prieto JH, García Fraile JA. Secuencias didácticas: aprendizaje y evaluación de competencias. México: Perason Educación de México; 2010

2. Héctor V. Los docentes en el sistema educativo cubano: análisis de su carrera, desarrollo profesional y evaluación de su desempeño. En Chile; 2006.

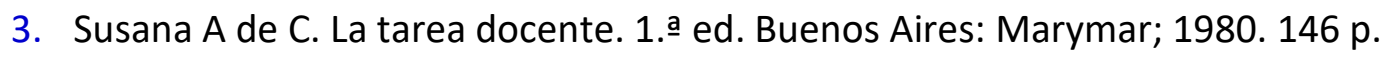

4. Plascencia JMO, Pintor SYV, Gutiérrez PMV, Pérez FKS, González MEC, Ávila BLC, et al. Revisión del proceso de evaluación docente por los estudiantes. 2011;42

5. Rodríguez H. Importancia de la formación de los docentes en las instituciones educativas [Internet]. Universidad Autónoma del Estado de Hidalgo. [citado 16 de abril de 2019]. Disponible en: https://www.uaeh.edu.mx/scige/boletin/huejutla/n9/e2.html

6. Valbuena Barrera, A. El Quirófano Como Ambiente De Aprendizaje. Sentido Y Significado Otorgado Por Estudiantes De Medicina [Internet] [citado 16 de abril de 2016]. Disponible en: http://www.bdigital.unal.edu.co/49375/1/19288830.2015.pdf

7. Martínez Abril C. Enfermería en Cirugía Ortopédica y Traumatología. [Internet] [citado 16 de abril de 2016]. Disponible en: http://media.axon.es/pdf/53681.pdf 